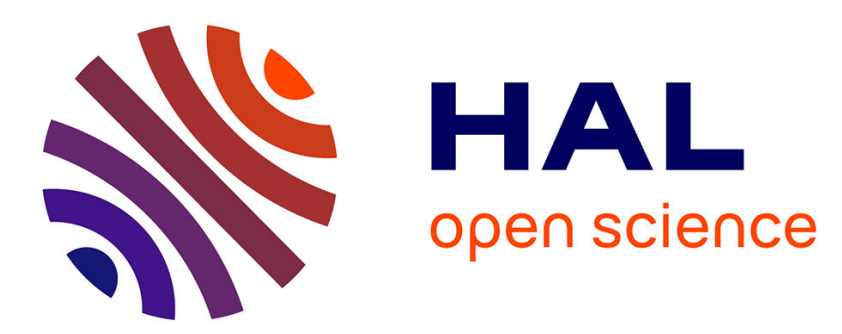

\title{
Fault diagnosis for nonlinear aircraft based on control-induced redundancy
}

Julien Marzat, Hélène Piet-Lahanier, Frédéric Damongeot, Eric Walter

\section{To cite this version:}

Julien Marzat, Hélène Piet-Lahanier, Frédéric Damongeot, Eric Walter. Fault diagnosis for nonlinear aircraft based on control-induced redundancy. IEEE Conference on Control and Fault-Tolerant Systems, SysTol'10, Oct 2010, Nice, France. 6 p. hal-00520805

\section{HAL Id: hal-00520805 \\ https://hal-centralesupelec.archives-ouvertes.fr/hal-00520805}

Submitted on 24 Sep 2010

HAL is a multi-disciplinary open access archive for the deposit and dissemination of scientific research documents, whether they are published or not. The documents may come from teaching and research institutions in France or abroad, or from public or private research centers.
L'archive ouverte pluridisciplinaire HAL, est destinée au dépôt et à la diffusion de documents scientifiques de niveau recherche, publiés ou non, émanant des établissements d'enseignement et de recherche français ou étrangers, des laboratoires publics ou privés. 


\title{
Fault diagnosis for nonlinear aircraft based on control-induced redundancy
}

\author{
Julien Marzat, Hélène Piet-Lahanier, Frédéric Damongeot, Éric Walter
}

\begin{abstract}
A Fault Detection and Isolation (FDI) method for a generic 3D aircraft is presented. The behavior of the aircraft is described by a nonlinear control-affine model, which is closed-loop controlled by a classical guidance, navigation and control (GNC) algorithm. The proposed FDI procedure exploits the redundancy induced by this control module, along with the accelerations measured by the Inertial Measurement Unit (IMU). Estimates of first-order derivatives of some state variables are thus readily available and allow one to estimate the control inputs as achieved by the actuators. Since the computed control input sent to the actuator is known, it is possible to use the discrepancy between these two elements as a residual indicative of faults. This strategy is successfully applied to the proposed aeronautical benchmark to detect and isolate actuator faults affecting simultaneously flight control surfaces and propulsion.

Index Terms - aerospace, aircraft, fault detection and isolation, fault diagnosis, nonlinear systems, system inversion.
\end{abstract}

\section{INTRODUCTION}

A reliability study [1] reports that about $80 \%$ of flight incidents concerning Unmanned Aerial Vehicles (UAV) are due to faults affecting propulsion, flight control surfaces or sensors. To allow the mission to carry on and to insure its safety, these faults should be detected early, before they lead to catastrophic failures. A classical way to tackle this problem is to use hardware redundancy, i.e., multiple devices performing the same function. However, this approach implies higher costs, lower autonomy and reduced payload, because of the additional weight, volume and power required. An interesting way to circumvent these difficulties is to rely on analytical redundancy, which exploits the relations between measured or estimated variables to detect possible dysfunctions of the system [2], [3].

The main FDI approaches, coming from different communities, are reviewed in [4], where the most promising methods for aeronautical systems are pointed out. Most model-based methods use a linear dynamical model of the aircraft (see, e.g., [5] or [6]). In aerospace, though, nonlinear models provide a more accurate representation of the vehicle complex behavior [7]. The immediate idea would be to linearize the model in order to be able to apply the familiar linear techniques, but the inaccuracy thus generated increases drastically non-detection and false-alarm rates [8]. Therefore, dedicated nonlinear methods should be investigated.

An interesting breakthrough has been achieved in a differential-geometric framework by De Persis and

J. Marzat, H. Piet-Lahanier and F. Damongeot are with ONERA-DPRS, Palaiseau, France, firstname.lastname@onera.fr

J. Marzat and É. Walter are with the Laboratoire des Signaux et Systmes (L2S), CNRS-SUPELEC-Univ-Paris-Sud, France, firstname.1astname@1ss.supelec.fr
Isidori [9]. This method extends the parity space approach [10] to nonlinear control-affine systems. It has been successfully applied to a two-dimensional aircraft model in [11]. Most FDI methods, including the latter, check whether the outputs of the system monitored are consistent with the inputs, given the model. Another interesting idea is inversion-based FDI, which uses the left-inverse of the nonlinear system (defined as in [12]) to check whether the expected inputs are consistent with the measured outputs [13]. However, all these approaches require the computation of successive derivatives of noisy and disturbed inputs and outputs (which can be numerically tricky), or model transformations that lack generality.

Aircraft are most often equipped with an Inertial Measurement Unit (IMU) that measures non-gravitational accelerations and angular rates. These quantities are then integrated by an Inertial Navigation System (INS) to estimate the entire state vector. They are derivatives of the velocity variables that are in the state vector. Building a left-inverse model through successive derivations of differential equations can thus be avoided. We propose to perform a direct inversion to estimate the achieved control inputs from available information. It is similar in spirit to that in [14], where acceleration is estimated instead. As the desired control inputs are known, consistency may then be checked between the estimated and expected control values.

This approach is illustrated on an aeronautical benchmark defined in [4], which is representative of a large panel of aerospace vehicles. The aircraft considered is equipped with classical, non-redundant sensors and actuators. Its threedimensional behavior is represented by a nonlinear controlaffine model described in Section II.

The practical application of the proposed FDI approach to this aeronautical benchmark is the focal point of this paper. A formal description of the method is available in [15]. The preliminary analytical computation is explained in Section III and simulation results are displayed in Section IV. They show that the fault diagnosis approach allows the detection, isolation and even identification of multiple actuator faults. Conclusions and perspectives are discussed in Section V.

\section{Aeronautical benchmark}

The vehicle considered is a surface-to-air missile on an interception mission. It is equipped with an IMU, coupled with an INS. The orientation of the vehicle is governed by three rudders, one per axis. The propulsion is regulated along the main axis of the missile, which is assumed cylindrical. The geometry of the aircraft in its body frame is displayed in Figure 1. These features are not specific to the case study 
and are shared by other applications, such as an UAV on an exploration mission. The description of the flight dynamics is stated below.

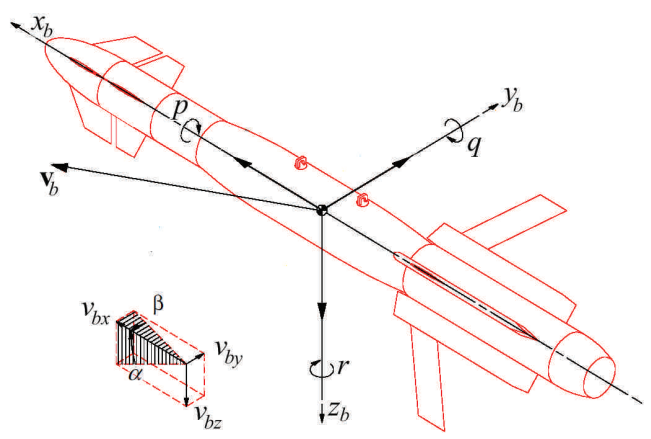

Fig. 1. Missile scheme in body frame

\section{A. Notation}

- $\mathbf{I}=\operatorname{diag}(a, b, b)$ is the inertia matrix of the system,

- $[x, y, z]$ is the position in the inertial frame,

- $\left[v_{\mathrm{bx}}, v_{\mathrm{by}}, v_{\mathrm{bz}}\right]$ is the speed in body coordinates,

- $\left[a_{\mathrm{bx}}, a_{\mathrm{by}}, a_{\mathrm{bz}}\right]$ is the non-gravitational acceleration in body coordinates,

- $[\varphi, \theta, \psi]$ is the orientation of the vehicle,

- $[p, q, r]$ is the angular velocity,

- $\left[\delta_{\mathrm{l}}, \delta_{\mathrm{m}}, \delta_{\mathrm{n}}\right]$ are the rudder deflection angles,

- $\eta$ is the propulsion rate,

- $Q=\frac{1}{2} \rho\left(v_{\mathrm{bx}}^{2}+v_{\mathrm{by}}^{2}+v_{\mathrm{bz}}^{2}\right)$ is the dynamic pressure,

- $\alpha=\arctan \left(\frac{v_{\mathrm{bz}}}{v_{\mathrm{bx}}}\right)$ is the angle of attack,

- $\beta=\arctan \left(\frac{v_{\mathrm{by}}}{v_{\mathrm{bx}}}\right)$ is the sideslip angle,

- $m$ is the aircraft mass,

- $f_{\min }$ and $f_{\max }$ are constants of the propulsion model,

- $s_{\text {ref }}$ and $l_{\text {ref }}$ are the reference surface and length,

- $c_{(.)}$are the aerodynamic coefficients, known piecewise continuous nonlinear functions of $(\alpha, \beta)$.

\section{B. Dynamics}

The force equation is

$$
\left[\begin{array}{c}
a_{\mathrm{bx}} \\
a_{\mathrm{by}} \\
a_{\mathrm{bz}}
\end{array}\right]=\left[\begin{array}{c}
\dot{v}_{\mathrm{bx}} \\
\dot{v}_{\mathrm{by}} \\
\dot{v}_{\mathrm{bz}}
\end{array}\right]+\left[\begin{array}{c}
p \\
q \\
r
\end{array}\right] \wedge\left[\begin{array}{c}
v_{\mathrm{bx}} \\
v_{\mathrm{by}} \\
v_{\mathrm{bz}}
\end{array}\right]=\frac{1}{m}\left(\mathbf{f}_{\mathrm{aero}}+\mathbf{f}_{\mathrm{g}}\right)
$$

where

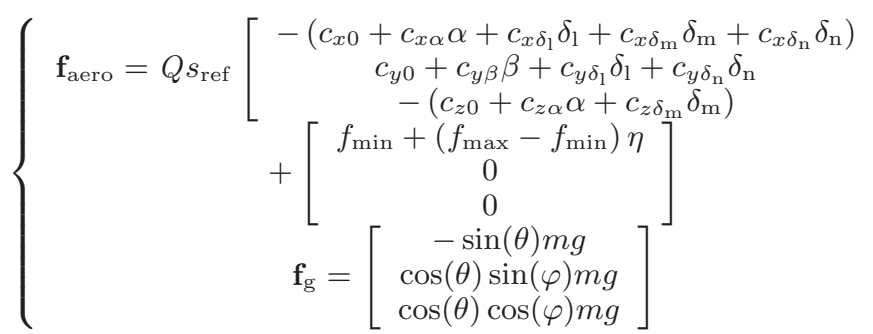

Since the inertia matrix is diagonal, the momentum equation is

$$
\left\{\begin{array}{c}
\dot{p}=\frac{Q s_{\mathrm{ref}} L}{a} \\
\dot{q}=\frac{1}{b}\left[Q s_{\mathrm{ref}} M-(a-b) p r\right] \\
\dot{r}=\frac{1}{b}\left[Q s_{\mathrm{ref}} N-(b-a) p q\right]
\end{array}\right.
$$

where

$$
\left\{\begin{array}{c}
L=c_{10}+c_{1 \beta} \beta+c_{1 \delta_{1}} \delta_{1}+c_{1 \delta_{\mathrm{n}}} \delta_{\mathrm{n}}+\frac{l_{\mathrm{ref}}}{\sqrt{v_{\mathrm{bx}}^{2}+v_{\mathrm{by}}^{2}+v_{\mathrm{bz}}^{2}}} c_{\mathrm{l}} p \\
M=c_{\mathrm{m} 0}+c_{\mathrm{m} \alpha} \alpha+c_{\mathrm{m} \delta_{\mathrm{m}}} \delta_{\mathrm{m}}+\frac{l_{\mathrm{ref}}}{\sqrt{v_{\mathrm{bx}}^{2}+v_{\mathrm{by}}^{2}+v_{\mathrm{bz}}^{2}}} c_{\mathrm{mq}} q \\
N=c_{\mathrm{n} 0}+c_{\mathrm{n} \beta} \beta+c_{\mathrm{n} \delta_{1}} \delta_{1}+c_{\mathrm{n} \delta_{\mathrm{n}}} \delta_{\mathrm{n}}+\frac{l_{\mathrm{ref}}}{\sqrt{v_{\mathrm{bx}}^{2}+v_{\mathrm{by}}^{2}+v_{\mathrm{bz}}^{2}}} c_{\mathrm{nr}} r
\end{array}\right.
$$

The angular dynamics is

$$
\left[\begin{array}{c}
\dot{\varphi} \\
\dot{\theta} \\
\dot{\psi}
\end{array}\right]=\left[\begin{array}{ccc}
1 & \sin \varphi \tan \theta & \cos \varphi \tan \theta \\
0 & \cos \varphi & -\sin \varphi \\
0 & \frac{\sin \varphi}{\cos \theta} & \frac{\cos \varphi}{\cos \theta}
\end{array}\right]\left[\begin{array}{l}
p \\
q \\
r
\end{array}\right]
$$

The model description should be completed with the coordinate transformation from the body frame to the inertial frame,

$$
\left[\begin{array}{c}
\dot{x} \\
\dot{y} \\
\dot{z}
\end{array}\right]=\left[\begin{array}{ccc}
\cos \psi \cos \theta & R_{\mathrm{b} 1} & R_{\mathrm{b} 2} \\
\sin \psi \cos \theta & R_{\mathrm{b} 3} & R_{\mathrm{b} 4} \\
-\sin \theta & \cos \theta \sin \varphi & \cos \theta \cos \varphi
\end{array}\right]\left[\begin{array}{c}
v_{\mathrm{bx}} \\
v_{\mathrm{by}} \\
v_{\mathrm{bz}}
\end{array}\right]_{(4)}
$$

where

$$
\begin{gathered}
R_{\mathrm{b} 1}=-\sin \psi \cos \varphi+\cos \psi \sin \theta \sin \varphi \\
R_{\mathrm{b} 2}=\sin \psi \sin \varphi+\cos \psi \sin \theta \cos \varphi \\
R_{\mathrm{b} 3}=\cos \psi \cos \varphi+\sin \psi \sin \theta \sin \varphi \\
R_{\mathrm{b} 4}=-\cos \psi \sin \varphi+\sin \psi \sin \theta \cos \varphi
\end{gathered}
$$

The state vector is $\mathbf{x}=\left[x, y, z, v_{\mathrm{bx}}, v_{\mathrm{by}}, v_{\mathrm{bz}}, p, q, r, \varphi, \theta, \psi\right]^{\mathrm{T}}$ and the input vector is $\mathbf{u}=\left[\delta_{1}, \delta_{\mathrm{m}}, \delta_{\mathrm{n}}, \eta,\right]^{\mathrm{T}}$. This complete control-affine nonlinear model has 12 state variables, which is highly classical in aerospace models. The complete model structure is obtained by gathering equations (1), (2), (3) and (4).

\section{Sensors and Guidance, Navigation and Control (GNC)}

The IMU measures the non-gravitational acceleration and angular velocity. The INS integrates these elements to estimate the position, velocity and orientation of the vehicle. The resulting output vector is $\mathbf{y}=\left[a_{\mathrm{bx}}, a_{\mathrm{by}}, a_{\mathrm{bz}}, p, q, r, x, y, z, v_{\mathrm{bx}}, v_{\mathrm{by}}, v_{\mathrm{bz}}, \varphi, \theta, \psi\right]^{\mathrm{T}} . \mathrm{Er}-$ rors affecting the measurements are usually modeled as biases, scale factors and noise. For example, the measurement of the yaw rate $r$ is expressed as $\tilde{r}=k_{r} r+b_{r}+w_{r}$ where $k_{r}$ is the scale factor, $b_{r}$ the bias and $w_{r}$ follows a zero-mean Gaussian distribution with standard deviation $\sigma_{r}$. These three parameters (for each sensor) are characteristic of the IMU and set in the simulated test case at typical values.

An interception guidance law is designed to drive the distance between missile and target to zero. The upper part of Figure 2 shows the guidance architecture, which is a closed-loop control using exogenous target information. The classical approach separately defines the guidance law and an autopilot that translates acceleration orders in actuator rotations. Further details concerning the GNC theory used in the benchmark can be found in [16].

\section{Faults}

We need to distinguish two types of control input vectors.

- The (known) control input vector as computed by the GNC module, $\mathbf{u}_{\mathrm{c}}=\left[\delta_{\mathrm{lc}}, \delta_{\mathrm{mc}}, \delta_{\mathrm{nc}}, \eta_{\mathrm{c}}\right]^{\mathrm{T}}$.

- The (unknown) control input vector as achieved by the actuators, $\mathbf{u}_{\mathrm{a}}=\left[\delta_{\mathrm{la}}, \delta_{\mathrm{ma}}, \delta_{\mathrm{na}}, \eta_{\mathrm{a}}\right]^{\mathrm{T}}$. 
Sensor faults may be modeled as unexpected variations in the uncertainty parameters of the IMU. We focus here on actuator faults, which result in discrepancies between $\mathbf{u}_{\mathrm{c}}$ and $\mathbf{u}_{\mathrm{a}}$. This means that the control input sent by the GNC module is not correctly implemented by the actuator. Actuators faults will be modeled as

$$
\mathbf{u}_{\mathrm{a}}=\sigma_{\mathrm{f}} \cdot k_{\mathrm{f}} \cdot \mathbf{u}_{\mathrm{c}}+\left(1-\sigma_{\mathrm{f}}\right) \cdot \mathbf{u}_{\mathrm{f}}
$$

where $\forall t>t_{\text {fault }}$, depending on the type of fault occurring, and where the parameters are such that

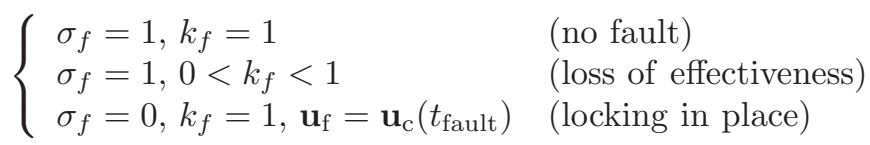

This fault model will be used to simulate two major faults affecting actuators, namely the loss of effectiveness of the propulsion and the locking in place of one rudder (or more).

\section{FAULT DIAGNOSIS}

\section{A. Principles}

The key idea is to use the control-affine structure of the system and the available measurements to extract directly an estimate of the achieved control input vector, without computing an inverse state-space model. Let $\widehat{\mathbf{u}}_{\mathrm{a}}$ be this estimate of $\mathbf{u}_{\mathrm{a}}$. Fault residuals are then obtained by comparing $\widehat{\mathbf{u}}_{\mathrm{a}}$ with $\mathbf{u}_{\mathrm{c}}$. The complete architecture of the system coupled with the fault diagnosis procedure is displayed in Figure 2.

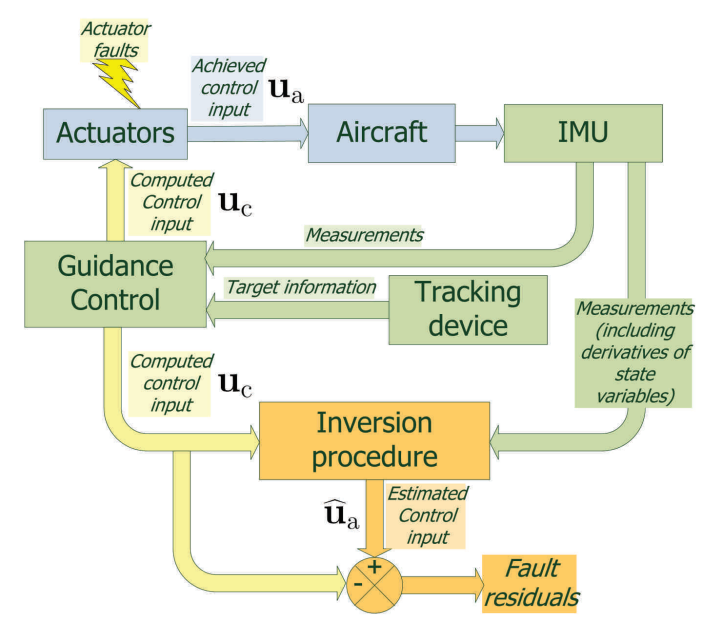

Fig. 2. Vehicle loop and residual generation

Many inversion procedures can be considered. We now present a possible approach to obtain expressions of $\widehat{\mathbf{u}}_{\mathrm{a}}$ that will lead to reliable fault detection, isolation and identification.

The first step is to select, from the entire state-space model described in Section II-B, state equations where the control inputs are the only unknown variables. In other words, these equations must contain only measured (or reliably estimated) state variables and their derivatives, and control inputs. The only group of equations fulfilling this requirements is that of the force equations (1). Indeed, there is no control input present in (3) and (4), so these equations are useless for the proposed FDI method. Equations of (2) are also excluded, because the variables $\dot{p}, \dot{q}, \dot{r}$ are not measured. For fault diagnosis, we thus consider only the force equation, whose only non-measured variables are the control inputs, which appear in an affine manner

$$
\left\{\begin{array}{c}
a_{\mathrm{bx}}=-\frac{Q s_{\mathrm{ref}}}{m}\left[c_{x 0}+c_{x a} \alpha+c_{x \delta_{1}} \delta_{\mathrm{l}}+c_{x \delta_{\mathrm{m}}} \delta_{\mathrm{m}}+c_{x \delta_{\mathrm{n}}} \delta_{\mathrm{n}}\right] \\
+\frac{1}{m}\left[f_{\min }+\left(f_{\max }-f_{\min }\right) \eta\right] \\
a_{\mathrm{by}}=\frac{Q s_{\mathrm{ref}}}{m}\left[c_{y 0}+c_{y b} \beta+c_{y \delta_{1}} \delta_{\mathrm{l}}+c_{y \delta_{\mathrm{n}}} \delta_{\mathrm{n}}\right] \\
a_{\mathrm{bz}}=\frac{Q s_{\mathrm{ref}}}{m}\left[c_{z 0}+c_{z a} \alpha+c_{z \delta_{\mathrm{m}}} \delta_{\mathrm{m}}\right]
\end{array}\right.
$$

It could be formalized as $\mathbf{f}=\mathbf{G u}$, or more explicitly

$$
\left[\begin{array}{l}
f_{1} \\
f_{2} \\
f_{3}
\end{array}\right]=\left[\begin{array}{cccc}
g_{11} & g_{12} & g_{13} & g_{14} \\
g_{21} & 0 & g_{23} & 0 \\
0 & g_{32} & 0 & 0
\end{array}\right]\left[\begin{array}{c}
\delta_{1} \\
\delta_{\mathrm{m}} \\
\delta_{\mathrm{n}} \\
\eta
\end{array}\right]
$$

where

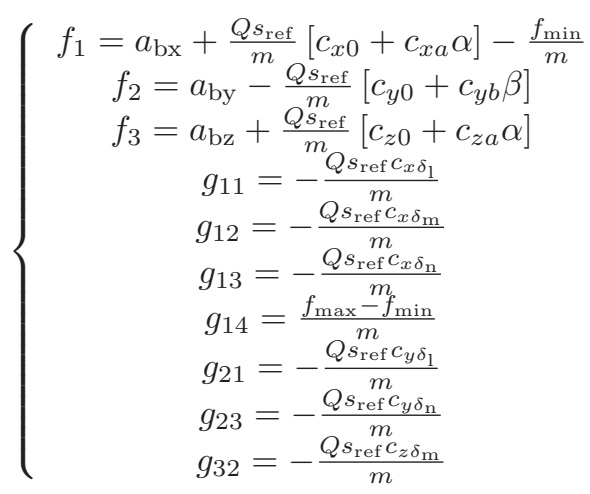

Note that these coefficients are all functions of the measurement vector $\mathbf{y}$, however this dependency will be omitted in what follows for the sake of simplicity.

\section{B. Direct residuals}

The idea of the estimation procedure is to consider separately each equation in (5). Using one of these equations, each control input involved can be estimated as a function of the measurements and the other control inputs. Substituting the computed values for the unknown values of the other inputs gives an estimate of this control input as achieved by the corresponding actuator. This direct procedure produces as many residuals as there are nonzero terms in $\mathbf{G}$ (seven here).

Consider, for example, the second equation $f_{2}=g_{21} \delta_{1}+$ $g_{23} \delta_{\mathrm{n}}$. It is possible to obtain estimates of $\delta_{\text {la }}$ and $\delta_{\text {na }}$ (roll and yaw angles achieved by the corresponding rudders) as

$$
\widehat{\delta}_{\mathrm{la}}=\frac{f_{2}-g_{23} \delta_{\mathrm{nc}}}{g_{21}}
$$

and

$$
\widehat{\delta}_{\text {na }}=\frac{f_{2}-g_{21} \delta_{\mathrm{lc}}}{g_{23}}
$$

These estimates are valid only when the corresponding denominators are nonzero. They are structurally so, but their value should nevertheless be checked and the residual not taken into account if the denominator is too close to zero. 
Residuals can now be generated by comparing the values obtained through (6) and (7) to the computed control inputs $\delta_{\mathrm{lc}}$ and $\delta_{\mathrm{nc}}$, provided by the control module, to get the residuals

$$
r_{21}=\widehat{\delta}_{\mathrm{la}}-\delta_{\mathrm{lc}}=\frac{f_{2}-g_{23} \delta_{\mathrm{nc}}}{g_{21}}-\delta_{\mathrm{lc}}
$$

and

$$
r_{23}=\widehat{\delta}_{\text {na }}-\delta_{\mathrm{nc}}=\frac{f_{2}-g_{21} \delta_{\mathrm{lc}}}{g_{23}}-\delta_{\mathrm{nc}}
$$

Five other residuals are obtained similarly from the first and third equations of (5).

\section{Sensitivity and robustness}

The sensitivity of the residuals to actuator faults should be analyzed, to ensure the possibility of isolation when multiple faults occur. If we consider the previously generated residual $r_{21}$ and replace $f_{2}$ by its expression from (5), we have

$$
\begin{aligned}
r_{21} & =\frac{g_{21} \delta_{\mathrm{la}}+g_{23} \delta_{\mathrm{na}}-g_{23} \delta_{\mathrm{nc}}}{g_{21}}-\delta_{\mathrm{lc}} \\
& =\left(\delta_{\mathrm{la}}-\delta_{\mathrm{lc}}\right)+\frac{g_{23}}{g_{21}}\left(\delta_{\mathrm{na}}-\delta_{\mathrm{nc}}\right)
\end{aligned}
$$

Therefore, if a fault affecting the roll rudder $\delta_{1}$ occurs and if there is no fault affecting the yaw rudder $\delta_{\text {n }}$ (i.e., $\delta_{\text {na }}=$ $\delta_{\text {nc }}$ ), then the residual $r_{21}$ measures the distance between $\delta_{\text {la }}$ and $\delta_{\mathrm{lc}}$ and directly identifies the error of angle affecting the roll rudder. On the other hand, if there is no fault affecting $\delta_{\mathrm{l}}$ and if a fault affecting $\delta_{\mathrm{n}}$ occurs, the residual will react to it but will not allow a direct identification of the fault because of the factor involved in (9). The fault on $\delta_{\mathrm{n}}$, if isolated, can nevertheless be identified by multiplying $r_{21}$ by $g_{21} / g_{23}$. The residual $r_{23}$ has the opposite features. This analysis makes it possible to fill in the first seven rows of Table I.

To get an idea of the robustness of the method to model uncertainty, let the actual equation for $f_{2}$ in (5) be $f_{2}=\left(g_{21}+\varepsilon_{1}\right) \delta_{1}+\left(g_{23}+\varepsilon_{2}\right) \delta_{\mathrm{n}}$, where $\varepsilon_{1}$ and $\varepsilon_{2}$ are small parameters (not necessarily constant). This represents model uncertainty on constant parameters (e.g., mass) and aerodynamic coefficients $c_{(\cdot)}$, which are partially unknown in practice. Injecting this expression for $f_{2}$ in (8) yields

$$
\begin{aligned}
& r_{21}=\frac{\left(g_{21}+\varepsilon_{1}\right) \delta_{\mathrm{la}}+\left(g_{23}+\varepsilon_{2}\right) \delta_{\mathrm{na}}-g_{23} \delta_{\mathrm{nc}}}{g_{21}}-\delta_{\mathrm{lc}} \\
& =\left(\delta_{\mathrm{la}}-\delta_{\mathrm{lc}}\right)+\frac{g_{23}}{g_{21}}\left(\delta_{\mathrm{na}}-\delta_{\mathrm{nc}}\right)+\frac{1}{g_{21}}\left(\varepsilon_{1} \delta_{\mathrm{la}}+\varepsilon_{2} \delta_{\mathrm{na}}\right) \\
& =\left(\delta_{\mathrm{la}}-\delta_{\mathrm{lc}}\right)+\frac{g_{23}}{g_{21}}\left(\delta_{\mathrm{na}}-\delta_{\mathrm{nc}}\right)+\Delta\left(\varepsilon_{1}, \varepsilon_{2}, \mathbf{u}_{\mathrm{a}}, \mathbf{y}\right)
\end{aligned}
$$

The residual is still sensitive to the two faults on $\delta_{\mathrm{l}}$ and $\delta_{\mathrm{n}}$ as explained in the sensitivity analysis, however an additional term $\Delta(\cdot)$ has appeared, in which

- $\varepsilon_{1}$ and $\varepsilon_{2}$ are small parameters. A bound on their value may be known or assumed;

- $\delta_{\text {la }}$ and $\delta_{\text {na }}$ are the unknown but bounded (due to physical saturation) control inputs as achieved by the actuators;

- the $g_{(\cdot)} s$ are known functions of the measurements.
The available information on these variables implies that upper and lower bounds on $\Delta(\cdot)$ are computable at each time step. If $\varepsilon_{1}$ and $\varepsilon_{2}$ are small enough, $\Delta$ should remain smaller than the size of the fault that needs to be detected and isolated. Nevertheless, identification of the numerical value of the fault in such conditions should be carried out with care. The same type of dependency to model uncertainty is observed with observer-based or parity space approaches as described, e.g., in [3] or in [17].

\section{Additional residuals}

The residuals obtained so far are functions of the available measurements and computed control inputs. Others may be obtained by substitution.

1) First substitution: The second equation in (5) contains only one control input, $\delta_{\mathrm{m}}$. It is then possible to obtain an estimator of this control input as a function of the measurements only,

$$
\widehat{\delta}_{\mathrm{m}}=\frac{f_{3}}{g_{32}}
$$

This expression can be injected into the residuals in place of the computed control input $\delta_{\mathrm{mc}}$. Consider, for example, the residual

$$
r_{11}=\frac{f_{1}-g_{12} \delta_{\mathrm{mc}}-g_{13} \delta_{\mathrm{nc}}-g_{14} \eta_{\mathrm{c}}}{g_{11}}-\delta_{\mathrm{lc}}
$$

and substitute $\widehat{\delta}_{\mathrm{m}}$ as given by (10) for $\delta_{\mathrm{mc}}$ to get the alternative residual

$$
\tilde{r}_{11}^{1}=\frac{f_{1}-g_{12} \frac{f_{3}}{g_{32}}-g_{13} \delta_{\mathrm{nc}}-g_{14} \eta_{\mathrm{c}}}{g_{11}}-\delta_{\mathrm{lc}}
$$

As shown in Section III-C, sensitivity to a given actuator fault is indicated by the presence of the corresponding computed control input in the residual. Here, $r_{11}$ is sensitive to faults affecting all the actuators while $\tilde{r}_{11}^{1}$ is insensitive to faults on $\delta_{\mathrm{m}}$. Four such residuals are obtained from the first equation of (5), and their sensitivity was analyzed as in Section III-C to complete Table I.

2) Second and third substitution: The second equation of (5) makes it possible to express $\delta_{\mathrm{l}}$ as a function of $\delta_{\mathrm{n}}$ and the measurements and reciprocally $\delta_{\mathrm{n}}$ as a function of $\delta_{\mathrm{l}}$ and the measurements, to get

$$
\widehat{\delta}_{\mathrm{l}}=\frac{f_{2}-g_{23} \delta_{\mathrm{nc}}}{g_{21}}
$$

and

$$
\widehat{\delta}_{\mathrm{n}}=\frac{f_{2}-g_{21} \delta_{\mathrm{lc}}}{g_{23}}
$$

Substituting $\widehat{\delta}_{\mathrm{l}}$ as given by (12) for $\delta_{\mathrm{lc}}$ in (11), one obtains the new residual

$$
\begin{aligned}
& \tilde{r}_{11}^{2}=\frac{f_{1}-g_{12} \delta_{\mathrm{mc}}-g_{13} \delta_{\mathrm{nc}}-g_{14} \eta_{\mathrm{c}}}{g_{11}}-\frac{f_{2}-g_{23} \delta_{\mathrm{nc}}}{g_{21}} \\
& =\frac{f_{1}-g_{12} \delta_{\mathrm{mc}}-g_{14} \eta_{\mathrm{c}}}{g_{11}}-\frac{f_{2}}{g_{21}}-\left(\frac{g_{13}}{g_{11}}-\frac{g_{23}}{g_{21}}\right) \delta_{\mathrm{nc}}
\end{aligned}
$$

To analyze the sensitivity of this new residual, $f_{1}$ and $f_{2}$ 
are replaced by their expression from (5) to get

$$
\begin{aligned}
\tilde{r}_{11}^{2}= & \frac{g_{12}}{g_{11}}\left(\delta_{\mathrm{ma}}-\delta_{\mathrm{mc}}\right)+\frac{g_{14}}{g_{11}}\left(\eta_{\mathrm{a}}-\eta_{\mathrm{c}}\right) \\
& +\left(\frac{g_{13}}{g_{11}}-\frac{g_{23}}{g_{21}}\right)\left(\delta_{\mathrm{na}}-\delta_{\mathrm{nc}}\right)
\end{aligned}
$$

This residual is thus insensitive to $\delta_{\mathrm{l}}$, which allows fault isolation between $\delta_{1}$ and $\delta_{\mathrm{n}}$. However, no direct fault identification is possible due to the functions involved. The other residuals $\tilde{r}_{12}^{2}, \tilde{r}_{13}^{2}, \tilde{r}_{14}^{2}$ will be sensitive to the same actuator faults but may allow further fault identification.

The same kind of substitution can be made with (13), producing one additional residual $\tilde{r}_{11}^{3}$ that will be sensitive to faults affecting $\delta_{\mathrm{l}}, \delta_{\mathrm{m}}$ and $\eta$ but not $\delta_{\mathrm{n}}$. Three other residuals could be obtained and will present sensitivity to the same actuator faults.

3) Fourth and fifth substitution: It is even possible to go further. As earlier, $\delta_{\mathrm{mc}}$ can be replaced by the estimate (10) in the residuals generated in the previous paragraph, which produces residuals $\tilde{r}_{11}^{4}$, which is sensitive to faults affecting only $\delta_{\mathrm{n}}$ and $\eta$, and $\tilde{r}_{11}^{5}$, which is sensitive to faults affecting only $\delta_{\mathrm{l}}$ and $\eta$. Table I sums up the sensitivity of all these residuals to the actuator faults.

TABLE I

FAULT SIGNATURES

\begin{tabular}{|l|c|c|c|c|}
\cline { 2 - 5 } \multicolumn{1}{l|}{} & $\delta_{\mathrm{l}}$ & $\delta_{\mathrm{m}}$ & $\delta_{\mathrm{n}}$ & $\eta$ \\
\hline$r_{11}$ & 1 & 1 & 1 & 1 \\
\hline$r_{12}$ & 1 & 1 & 1 & 1 \\
\hline$r_{13}$ & 1 & 1 & 1 & 1 \\
\hline$r_{14}$ & 1 & 1 & 1 & 1 \\
\hline$r_{21}$ & 1 & 0 & 1 & 0 \\
\hline$r_{23}$ & 1 & 0 & 1 & 0 \\
\hline$r_{32}$ & 0 & 1 & 0 & 0 \\
\hline$\tilde{r}_{11}^{1}$ & 1 & 0 & 1 & 1 \\
\hline$\tilde{r}_{11}^{2}$ & 0 & 1 & 1 & 1 \\
\hline$\tilde{r}_{11}^{3}$ & 1 & 1 & 0 & 1 \\
\hline$\tilde{r}_{11}^{4}$ & 0 & 0 & 1 & 1 \\
\hline$\tilde{r}_{11}^{5}$ & 1 & 0 & 0 & 1 \\
\hline
\end{tabular}

Notice that the fault signature (the Boolean column) of each actuator in Table $I$ is unique. This means that fault detection and isolation is possible even when multiple faults occur successively or simultaneously. It has also been shown that fault identification may be possible if only one fault to whom the residual is sensitive occurs at a time.

\section{Simulation RESUlts}

The aeronautical benchmark (cf. Section II) is simulated with a time step of $0.01 \mathrm{~s}$. Uncertainty parameters affecting IMU measurements are tuned to typical values, as mentioned in Section II-C. The IMU is also assumed to suffer a delay of two time steps. Three fault scenarios are defined, a selection of residuals being displayed for each of them (cf. Table II). The corresponding 3D trajectories are presented in Figure 3.

TABLE II

FAULT SCENARIOS

\begin{tabular}{|c|c|c|}
\hline Scenario & Faults & Residuals displayed \\
\hline \hline 1 & $\left\{\begin{array}{c}\text { loss of } 25 \% \text { of } \eta \text { at } 30 \mathrm{~s} \\
\delta_{\mathrm{m}} \text { locked at } 30 \mathrm{~s} \\
\text { loss of } 25 \% \text { of } \eta \text { at } 50 \mathrm{~s}\end{array}\right.$ & $r_{14}, r_{12}, r_{23}$ \\
\hline 3 & $\left\{\begin{array}{c}\text { loss of } 50 \% \text { of } \eta \text { at } 20 \mathrm{~s} \\
\delta_{\mathrm{m}} \text { locked at } 45 \mathrm{~s} \\
\delta_{\mathrm{n}} \text { locked at } 30 \mathrm{~s}\end{array}\right.$ & $r_{23}, r_{32}, \tilde{r}_{11}^{4}, \tilde{r}_{14}^{5}$ \\
\hline
\end{tabular}

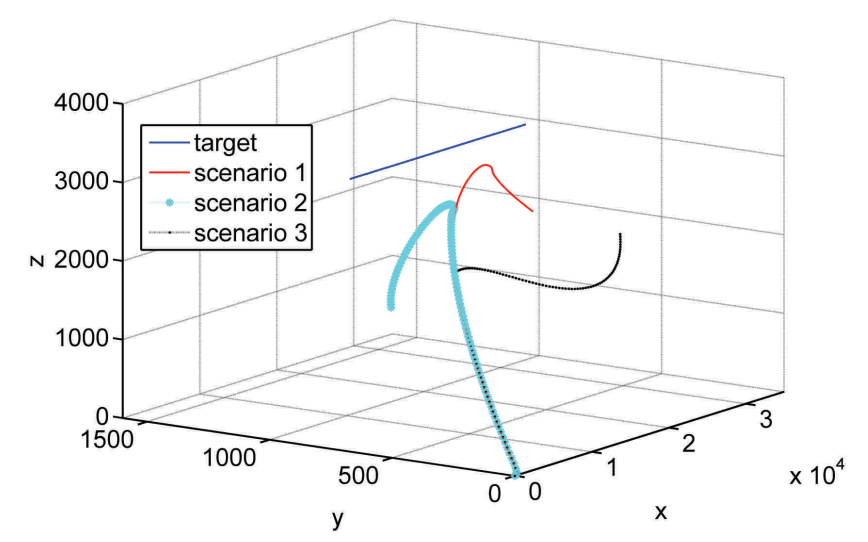

Fig. 3. Failed-interception trajectories

\section{A. Scenario 1 (see Figure 4)}

The $25 \%$ propulsion loss is identifiable with $r_{14}$. The residual $r_{12}$ allows the detection of the fault but is not tuned to identify it, while $r_{23}$ is insensitive to it. This abrupt fault can be detected with a very short delay.
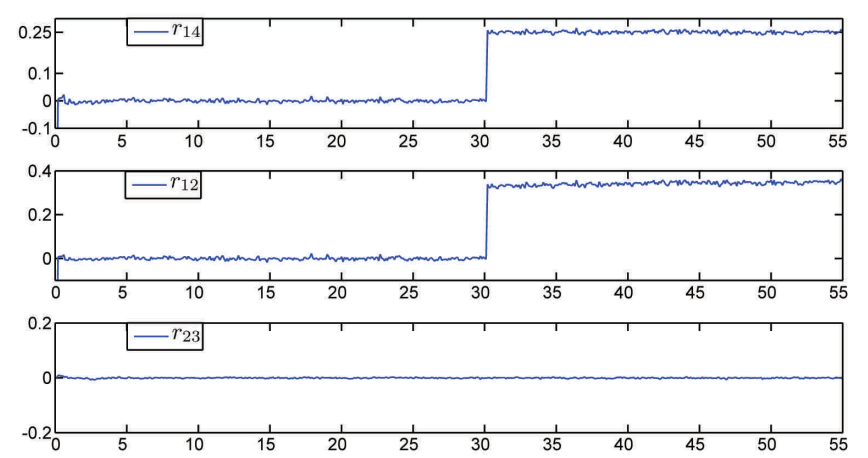

Fig. 4. Scenario 1

\section{B. Scenario 2 (see Figure 5)}

The residuals $r_{32}$ and $\tilde{r}_{14}^{1}$ respectively allow the detection and identification of faults on $\delta_{\mathrm{m}}$ and $\eta$. The residual $r_{21}$ is insensitive to both faults, ensuring isolation with respect to 
faults on the two other actuators. The incipient character of the fault affecting $\delta_{\mathrm{m}}$ will imply a delay in its detection and identification.
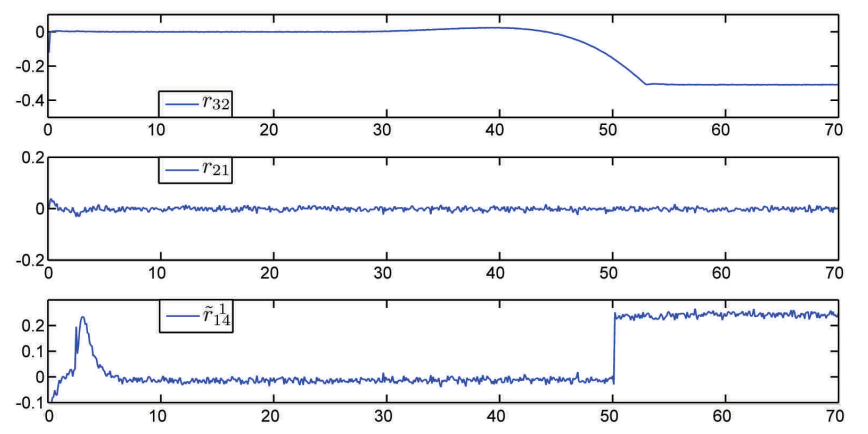

Fig. 5. Scenario 2

\section{Scenario 3 (see Figure 6)}

The residuals $r_{23}, r_{32}$ and $\tilde{r}_{14}^{5}$ are respectively sensitive to faults on $\delta_{\mathrm{n}}, \delta_{\mathrm{m}}$ and $\eta$. The residual $\tilde{r}_{11}^{4}$ reacts to both faults on propulsion and the yaw rudder.

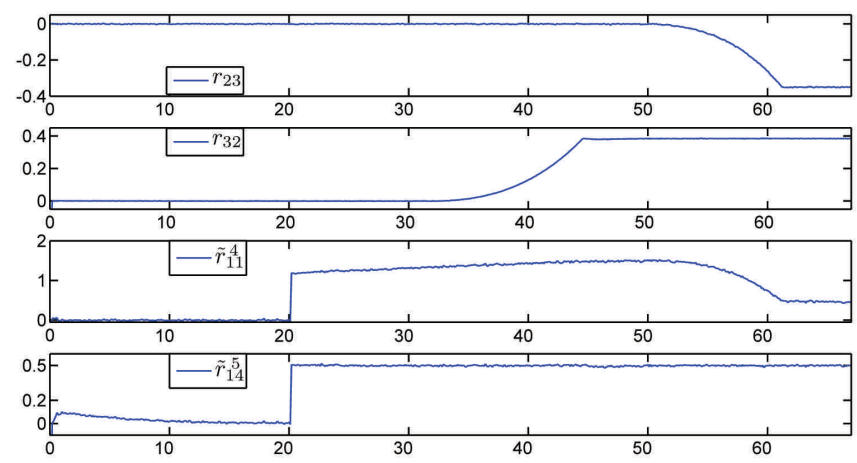

Fig. 6. Scenario 3

Note that, through this FDI procedure, the detection, isolation and identification of faults affecting three actuators (out of four) simultaneously is feasible, using only the three force equations.

\section{CONCLUSIONS AND PERSPECTIVES}

This paper was dedicated to the application to an aeronautical test-case of the nonlinear diagnosis method that has been formally presented in [15]. The major assumptions are that the behavior of the system can be reliably represented by a nonlinear control-affine model, and that an IMU (or any other device measuring acceleration) is available. If these assumptions are valid, then the force equation can be used to compute fault residuals. An inversion procedure to extract the achieved control inputs from this model has been presented. Residuals are obtained by comparing the estimates of the control inputs achieved by the actuators with the computed ones provided by the control module. Substitutions are then carried out to eliminate control inputs from the residuals. Fault detection and isolation of multiple faults affecting all the actuators has been shown to be feasible on the generic six-degrees-of-freedom aeronautical benchmark proposed, in the presence of realistic measurement uncertainty.

This paper was focused on the description of the residual generation procedure, and the residual analysis strategy remains to be chosen. Statistical tests and adaptive thresholds are interesting contenders. Sensor faults and robustness regarding disturbances (such as wind) should also be addressed.

Finally, the proposed method should be analyzed with respect to performance indices such as non-detection and false-alarm rates, detection delays and computational complexity. A framework to conduct as objectively as possible this rating and comparison with other FDI approaches should be designed.

\section{REFERENCES}

[1] R. Schaefer, "Unmanned aerial vehicle reliability study," Office of the Secretary of Defense, Washington, DC, 2003.

[2] R. J. Patton, "Fault detection and diagnosis in aerospace systems using analytical redundancy," Computing \& Control Engineering Journal, vol. 2, no. 3, pp. 127-136, 1991.

[3] P. M. Frank, "Fault diagnosis in dynamic systems using analytical and knowledge-based redundancy - a survey and some new results," Automatica, vol. 26, no. 3, pp. 459-474, 1990.

[4] J. Marzat, H. Piet-Lahanier, F. Damongeot, and E. Walter, "Autonomous fault diagnosis: State of the art and aeronautical benchmark," in Proceedings of the 3rd European Conference for Aero-Space Sciences, Versailles, France, 2009.

[5] R. J. Patton, F. J. Uppal, S. Simani, and B. Polle, "Robust FDI applied to thruster faults of a satellite system," Control Engineering Practice, 2009, doi:10.1016/j.conengprac.2009.04.011.

[6] G. Ducard and H. P. Geering, "Efficient nonlinear actuator fault detection and isolation system for unmanned aerial vehicles," Journal of Guidance Control and Dynamics, vol. 31, no. 1, pp. 225-237, 2008.

[7] J. Bokor and Z. Szabó, "Fault detection and isolation in nonlinear systems," Annual Reviews in Control, vol. 33, no. 2, pp. 113-123, 2009.

[8] M. Witczak, Modelling and Estimation Strategies for Fault Diagnosis of Non-Linear Systems: From Analytical to Soft Computing Approaches. Springer-Verlag, Berlin-Heidelberg, 2007.

[9] C. De Persis and A. Isidori, "A geometric approach to nonlinear fault detection and isolation," IEEE Transactions on Automatic Control, vol. 46, no. 6, pp. 853-865, 2001.

[10] E. Y. Chow and A. S. Willsky, "Analytical redundancy and the design of robust failure detection systems," IEEE Transactions on Automatic Control, vol. 29, no. 7, pp. 603-614, 1984.

[11] N. Meskin, T. Jiang, E. Sobhani, K. Khorasani, and C. A. Rabbath, "Nonlinear geometric approach to fault detection and isolation in an aircraft nonlinear longitudinal model," in Proceedings of the American Control Conference, New York, 2007, pp. 5771-5776.

[12] R. Hirschorn, "Invertibility of multivariable nonlinear control systems," IEEE Transactions on Automatic Control, vol. 24, no. 6, pp. 855-865, 1979.

[13] A. Edelmayer, J. Bokor, Z. Szabo, and F. Szigeti, "Input reconstruction by means of system inversion: A geometric approach to fault detection and isolation in nonlinear systems," International Journal of Applied Mathematics and Computer Science, vol. 14, no. 2, pp. 189-200, 2004.

[14] A. J. Ostroff and B. J. Bacon, "Force and moment approach for achievable dynamics using nonlinear dynamic inversion," in AIAA Guidance, Navigation, and Control Conference, Portland, Oregon, 1999, pp. 424-434.

[15] J. Marzat, H. Piet-Lahanier, F. Damongeot, and E. Walter, "Nonlinear FDI based on state derivatives, as provided by inertial measurement units," in Proceedings of the 8th IFAC Symposium on Nonlinear Control Systems NOLCOS'2010, Bologna, Italy, 2010.

[16] N. A. Shneydor, Missile Guidance and Pursuit: Kinematics, Dynamics \& Control. Horwood Publishing Limited, Chichester, 1998.

[17] J. J. Gertler, "Analytical redundancy methods in fault detection and isolation," in Proceedings of the 7th IFAC Symposium on Fault Detection, Supervision and Safety of Technical Processes, SAFEPROCESS 2009, Barcelona, Spain, vol. 1, 1991, pp. 9-21. 